\section{Electrospinning-based high-sensitive PVDF-TrFE nanofibre sensor with sensitivity dependence on pore diameter}

\author{
Fazle Kibria ${ }^{1}{ }^{*}$, Wahida Rahman ${ }^{2}$ and \\ Sankar Narayan Patra ${ }^{1}$ \\ ${ }^{1}$ Department of Instrumentation Science, and \\ ${ }^{2}$ Organic Nano-Piezoelectric Device Laboratory, \\ Department of Physics, Jadavpur University, Kolkata 700 032, India
}

Electrospinning-based nanofibre sensor of polyvinylidene fluoride-co-trifluoroethylene (PVDF-TrFE) was fabricated with versatile pore diameter, shape and size for assessment to be used in a wide range of biomedical applications. In this study, nanofibre film of PVDF-TrFE copolymers along with a sandwich of aluminium and copper acts as an electrode of the sensor, exhibiting strong acoustic to electricity conversion ability within the audible frequency range $20 \mathrm{~Hz}-$ $20 \mathrm{kHz}$. Sensitivity of the sensor was observed to effectively change from 0.5 to $12 \mathrm{VPa}^{-1}$ with change in pore diameter from 220 to $280 \mathrm{~nm}$ of the nanofibres. Besides, cardiac response within the frequency range $0.05-120 \mathrm{~Hz}$ at $0 \mathrm{~dB}$ was detected using this sensor with a sensitivity of $10 \mathrm{mV}$. The fabricated polymer sensor of $250 \mathrm{~nm}$ fibre pore diameter exhibited sensitivity as high as $10 \mathrm{VPa}^{-1}$ at $120 \mathrm{~Hz}$ incident frequency of audible sound under a specially arranged sensing assembly, where convergent sound directly impacted on the nanofibre layer. The high-sensitive materials can make a significant contribution in cardiac and audiometric device fabrication.

Keywords: Biomedical applications, cardiac and audiometric devices, electrospinning sensors, nanofibres, pore diameter.

FASTER development of electroactive polymers (EAPs) to fabricate artificial muscles and organs; smart materials for vibration and noise control; sensors and actuators for robotics application, etc. attracted the researcher to work in this field ${ }^{1}$. The function of the polymer in such applications is to convert acoustic energy into electricity. Polymers have many advantages such as transparency, flexibility, lightweight, easy processing and low cost in contrast to piezoceramic and magnetostrictive actuator materials ${ }^{2}$. Polymeric materials exhibit the properties of large-dimensional change without fatigue and robustness. Traditional piezoelectric EAPs exhibit low strain, low elastic energy density and low electromechanical conversion efficiency, which limit their applications in comparison with piezoelectric ceramics ${ }^{3}$. Therefore, the major challenges are to significantly increase and improve the

\footnotetext{
*For correspondence. (e-mail: jeemey@gmail.co.in)
}

electromechanical responses by achieving high strain capability, high elastic power density and high electromechanical conversion efficiency of EAPs for a broad range of applications ${ }^{4}$. Recent studies have revealed efforts to explore different new approaches to enhance the performance of existing polymers ${ }^{5}$. High electrostrictive strain with higher elastic modulus and longer load capability has been exhibited in a study on high-energy electron irradiated polyvinylidene fluoride-trifluoroethylene (PVDF-TrFE) copolymer ${ }^{6}$. Acoustic-based piezoelectric polymer sensors have high sensitivity and can be processed into wide geometries ${ }^{7}$. Nevertheless, almost all piezoelectric polymers are inevitably processed using the tedious method of mechanical extending and high-voltage poling at an enhanced temperature to attain piezoelectricity before making transducers ${ }^{8}$, which are energy-consuming and high-cost. Significantly, piezoelectricity of electrospinning nanofibres has been reported recently ${ }^{9-12}$. PVDF-TrFE copolymer at 30\% TrFE molar ratio readily developed $\beta$-crystal ${ }^{13-15}$ with exceptionally high capacitance in nano-Farad order ${ }^{16-19}$. This copolymer is mundane for its ferroelectric property that transfigures to paraelectric crystal with escalating temperature, otherwise Curie transition appear at $73^{\circ} \mathrm{C}$ (refs $20-23$ ). The developed piezoelectric polymer is classified as technically paramount in nature, having strong piezoelectricity without stretching and poling treatments ${ }^{24-27}$, with massive piezoelectric coefficient and minimal dielectric loss ${ }^{28-30}$. The copolymers have advantages of flexibility and moderate acoustic impedance, making them competitive for various sophisticated applications such as interaction of ultrasound with organic medium ${ }^{31-33}$, energy generation from mechanical stress ${ }^{34-36}$ and human movements as compared to piezoelectric ceramics ${ }^{37-39}$. Piezoelectric nanofibers demonstrate great potential as mechanical sensing elements and energy generators ${ }^{40,41}$. The voltage output of inconsistently aligned nanofibres under compression or bending is greater than in piezoelectric PVDF films. However, there are limited studies to date on the acoustic to electricity conversion of polymer nanofibres.

In this study nanofibres of PVDF-TrFE with different pore diameter, size, and shape were fabricated by electrospinning, which shows excellent acoustic to electricity conversion ability under different input signals. Metal electrodes enable output electricity by creating a potential difference between the two surfaces of the sensor. Moreover, polydimethylsiloxane (PDMS) wrapping will attract conductive ions towards the surface creating electrical charge polarization on the very site of the surfaces subjected to enhanced potential difference between them. The fabricated sensor and specially arranged sensing assembly make the incident divergent beam into an approximately convergent beam of longitudinal sound waves, which directly impact the nanofibres layer through a narrow hole of the cylinder. The device is capable of 
detecting low-frequency cardiac sound with sensitivity as high as $10 \mathrm{mVPa}^{-1}$. In comparison to the sensor device made of a piezoelectric PVDF, this film offers more sensitivity. It can precisely distinguish acoustic waves emitted from different acoustic sources of known frequencies within the normal audible region. The device shows a change in sensitivity under the application of normal audible conversation frequency within $20 \mathrm{~Hz}-20 \mathrm{kHz}$ with pressure level of $100 \mathrm{~dB}$ for different pore diameter of the nanofibres and size of the sensor, which is a significant outcome of this experimental study.

In this study, material pellets of PVDF $\left(\mathrm{CH}_{2} \mathrm{CF}_{2}\right)_{n}$ in powder form with vapour pressure $155 \mathrm{~mm}$ of $\mathrm{Hg}$ at normal temperature $\left(32^{\circ} \mathrm{C}\right)$ and molecular weight $\sim 530,000$; ready-to-use aqueous acetone $\left[\left(\mathrm{CH}_{3}\right)_{2}-\mathrm{CH}-\mathrm{OH}\right]$ solution of above $99 \%$ purity; methyl ethyl ketone solvent (MEK: $\mathrm{C}_{4} \mathrm{H}_{8} \mathrm{O}$ ) of specific gravity 0.8 , molecular weight 72.1 along with molar density $0.8 \mathrm{~g} / \mathrm{cm}^{3}$ and TrFE copolymer Solvene ${ }^{\circledR} 200 / \mathrm{P} 400\left[\left(\mathrm{C}_{2} \mathrm{H}_{2} \mathrm{~F}_{2}\right) \mathrm{n}\left(\mathrm{C}_{2} \mathrm{HF}_{3}\right) \mathrm{m}\right]$ in powder form with melt flow index $0.2-1.5$, average molecular weight 400 , piezoelectric coefficient $\left(d_{33}\right)-23 \mathrm{pC} / \mathrm{N}$ at $110 \mathrm{~Hz}$ with TrFE, $30 \mathrm{~mol} \%$ plus VDF, $70 \mathrm{~mol} \%$ mixture were used (Sigma-Aldrich Chemical Co, USA).

Off-the-rack PVDF-TrFE with a molar ratio of $70: 30$ (Sigma-Aldrich Chemical Co) in powder form, was dissolved with MEK in $12 \mathrm{wt} \%$. The solution was muddled for continuous $3 \mathrm{~h}$ using a magnetic stirrer at $120^{\circ} \mathrm{C}$ to ensure complete dissolution of the powder. Quantitatively $1.8 \mathrm{~g}$ of the powder was mixed with $15 \mathrm{ml}$ of MEK. The solution was sonicated for $15 \mathrm{~h}$ under electro-spinning primed. An automatic electrostatic-based system (Bruker make) was used to contrive nanofibre webs through Taylor cone formation and electrostatic repulsion at controlled atmospheric conditions of $27^{\circ} \mathrm{C}$ room temperature and $45 \%$ humidity. The prepared polymer solutions were poured into a $20 \mathrm{ml}$ syringe with stainless-steel needle (inner diameter: $0.85 \mathrm{~mm}$ ) attachment. Electrically earthened collector plate of the production unit was enclosed with aluminium foil and located $14 \mathrm{~cm}$ away from the needle tip. A high DC voltage of $8 \mathrm{kV}$ was applied between the needle tip and copper plate, while the infusion pace was set to $4 \mathrm{ml} / \mathrm{h}$. The system at $32^{\circ} \mathrm{C}$ temperature and $49 \%$ humidity under closed condition of the instrument took approximately $4 \mathrm{~h}$ to complete the process. The electrospun nanofibres were then dried for a few minutes before removing from the collector. Finally, freestanding flexible films of congested nano-webs on aluminium and copper foils were obtained.

The nanofibre film obtained from electrospinning was cut into pieces of dimension $10 \times 20 \mathrm{~mm}^{2}$ to fabricate the sensor. The acoustic sensor was prepared by sandwiching a piece of nanofibre film with thin metallic foil (thickness $\approx 0.01 \mathrm{~cm}$ ) as electrode and transparent coating of PDMS (thickness $80 \mathrm{~mm}$ ) membrane. Aluminium present on one veneer of the nanofibre film acts as an electrode of the sensor. On the other surface of the sensor, copper foil clamped on the film acts as a second electrode. Singlegauge copper wires were connected to both the electrodes to transmit the output response of the sensor. Two types of sensors were fabricated: aluminium-aluminium electrode-based sensor and aluminum-copper electrode-based sensor. Some of them were wrapped with fine layers of PDMS. Figure $1 a$ and $b$ represent solution preparation process for PVDF-TrFE and electrospinning process respectively. Figure $1 c$ shows a physically obtained PDMS-wrapped sensor and Figure $1 d$ shows different layers of the fabricated sensor.

The surface morphology of the nanofibres was observed under an elevated resolution scanning electron microscope (FE-SEM Zeiss). SEM images reveal the surface morphology, particle homogeneity and external atomic arrangement of the copolymer nanofibre web grown on the $0.01 \mathrm{~cm}$ thin aluminium foil. Photomicrographs were taken at different accelerating voltages from 5 to $20 \mathrm{KV}$ with alternative magnification ranging from $500 \mathrm{X}$ to $50 \mathrm{kX}$ for the characterization of microscopic structures of the nanofibre sensor. The porous surface geometry of the nanofibres indicates TrFE doping with PVDF. SEM study of the electrospun fibres showed no significant morphological variations in this particular PVDF/ TrFE ratio. However, depending on the concentration of the solution the porosity of the fibre changes; the surface of some fibres is smooth, whereas others show rough and/or no porosity (Figure $2 a-d$ ).

Experimental observations of electrospinning nanofibre film of PVDF-TrFE and PVDF alone were implemented using X-ray diffraction (XRD, Rigaku 800, table-top model) at $2 \theta$ versus intensity with $\theta$ value varying from $30^{\circ}$ to $90^{\circ}$ and scanning speed of $4 \mathrm{rpm}$ at $40 \mathrm{kV}$ and $40 \mathrm{~mA}$. Figure $3 a$ and $b$ shows the observed XRD pattern along with the Gaussian profile and full width at half maximum (FWHM) of the fabricated films.

Fourier-transform infrared reflection (FTIR) spectroscopic analysis in attenuated total reflection (ATR) mode (Bruker Optics) was performed over the range 700$1500 \mathrm{~cm}^{-1}$ with a resolution of $2 \mathrm{~cm}^{-1} ; 118$ scans were captured from initial temperature of $32^{\circ} \mathrm{C}$ to $150^{\circ} \mathrm{C}$ in heating phase and an analogous experiment was also performed for the cooling phase in reverse direction of temperature. Figure $4 a$ shows the FTIR plot of PVDF-TrFE nanofibre film for the heating cycle, whereas Figure $4 b$ shows a combined plot of the film and the intersection point of the two cycles.

A special arrangement was assembled to facilitate the conversion of divergent incident acoustic sound which is likely to be convergent as a electromagnetic beams, and impact on the nanofibre sensor directly. Figure 5 shows the set-up for measuring the sensing property. A sound system having provision to emit sound waves of different frequencies along with a metallic cylinder was used to converge the sound source. One end of the metallic cylinder was attached to the device to prevent air gap. To 


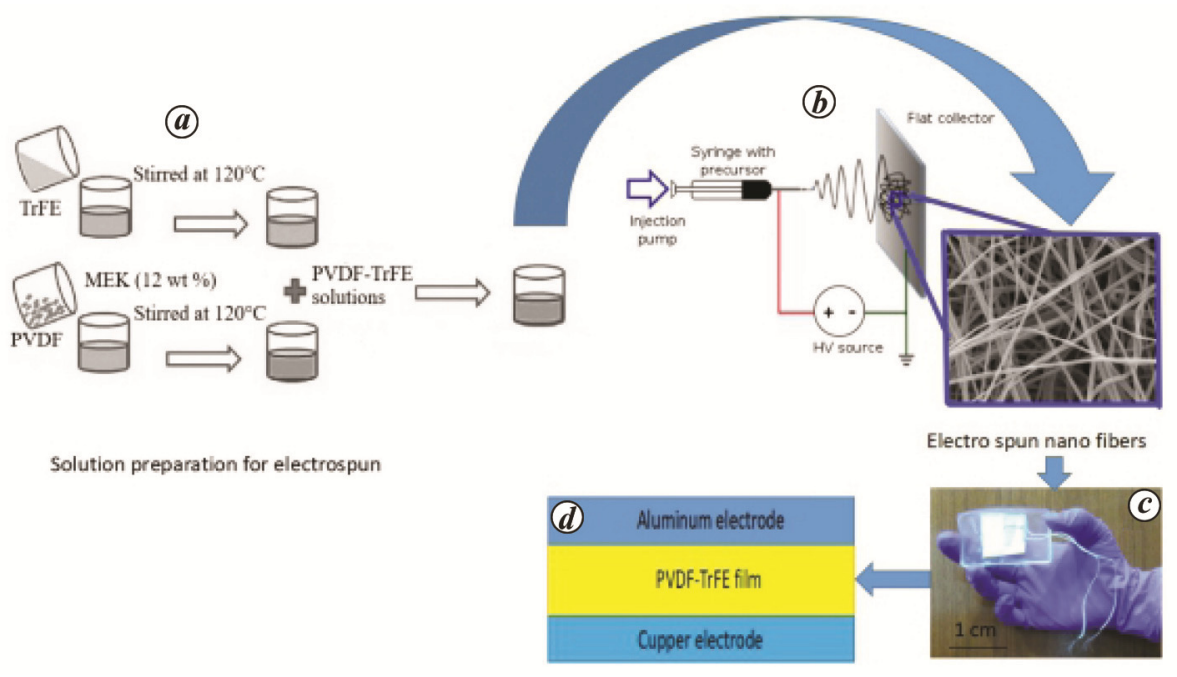

Figure 1. Fabrication hierarchy of polyvinylidene fluoride-co-trifluoroethylene (PVDF-TrFE) polymer sensor. $\boldsymbol{a}$, Preparation of PVDF-TrFE solution for electrospinning. $\boldsymbol{b}$, Electrospinning process for nanofibres production by highvoltage annealing. $c$, Physically obtained polydimethylsiloxane (PDMS) wrapped PVDF-TrFE acoustic sensor (scale bar: $1 \mathrm{~cm}) . \boldsymbol{d}$, Layers indication of the sensor.
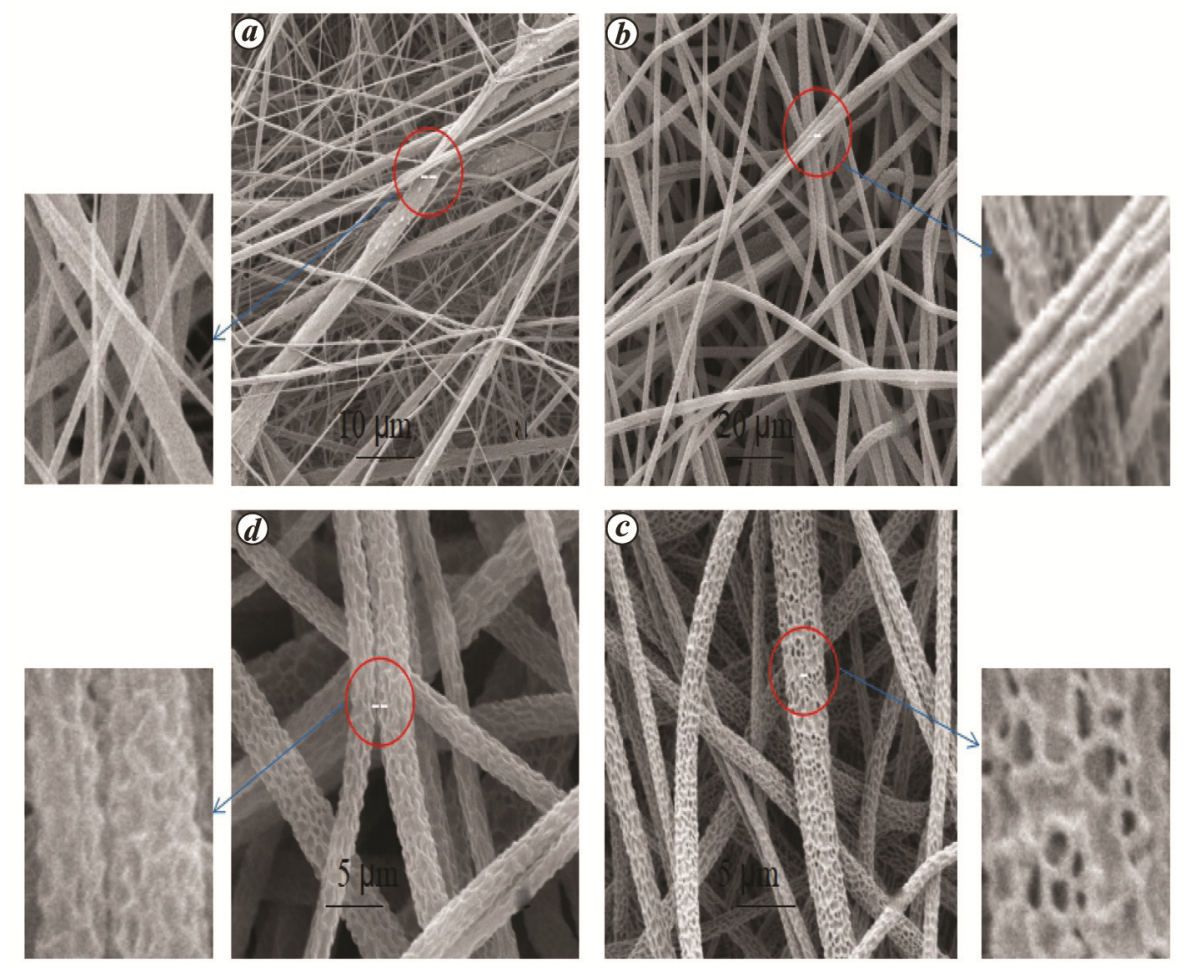

Figure 2. Scanning electron microscope (SEM) images of PVDF-TrFE electrospun fibres. $\boldsymbol{a}$, Fibres having no porosity on the surface (scale bar: $10 \mu \mathrm{m}$ ). $\boldsymbol{b}$, Smooth fibre surface with small pores (scale bar: $20 \mu \mathrm{m}$ ). $\boldsymbol{c}$, Rough fibre surface with large pore size (scale bar: $5 \mu \mathrm{m}$ ). $\boldsymbol{d}$, Smooth fibre surface with large pores (scale bar: $5 \mu \mathrm{m}$ ).

avoid signal interference and external acoustic influence on the testing equipment, all other acoustic sources were kept in complete shutdown mode. The testing equipment and sensor devices were fixed in a rigid and firm condition with an adjustable frame. In this way, unwanted influence from the background, supporting base and the total arrangement had a negligible contribution to the signal output of the sensor device even at very low acoustic signals. The sensors were tested using a commercial PC suite speaker (Logitech) as a sound source. The sound frequency was controlled by a computer user in controller mode. The same PC suite base system with Lab view facility was used to view and record the output. 

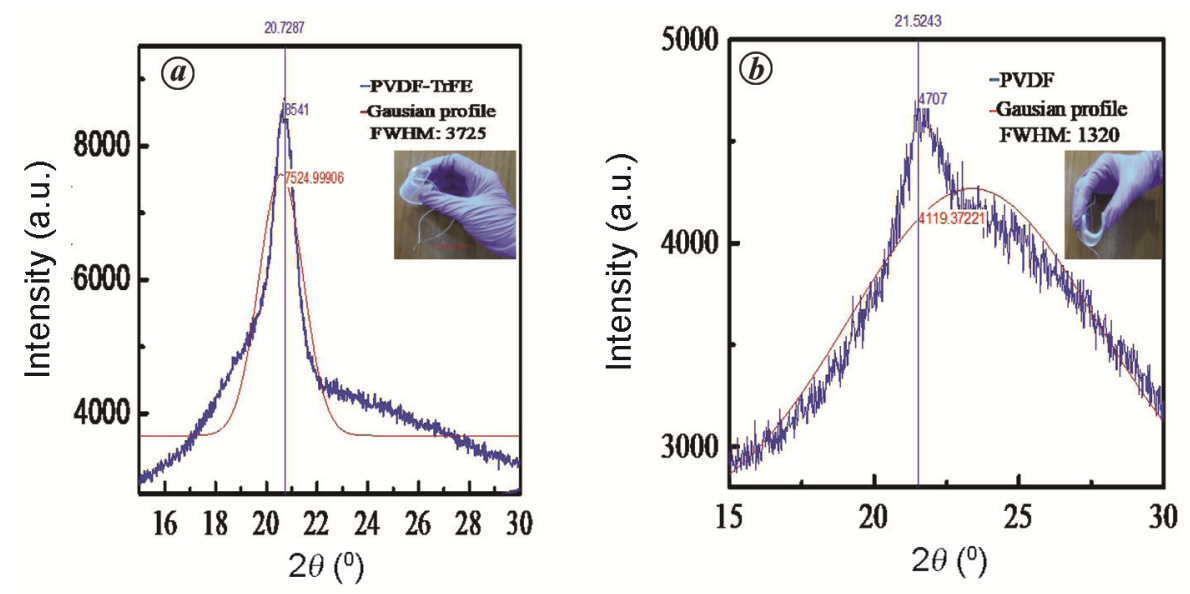

Figure 3. X-ray diffraction pattern with Gaussian profile of (a) PVDF-TrFE at full width at half maximum (FWHM) 3725 and (b) PVDF at FWHM 1320.
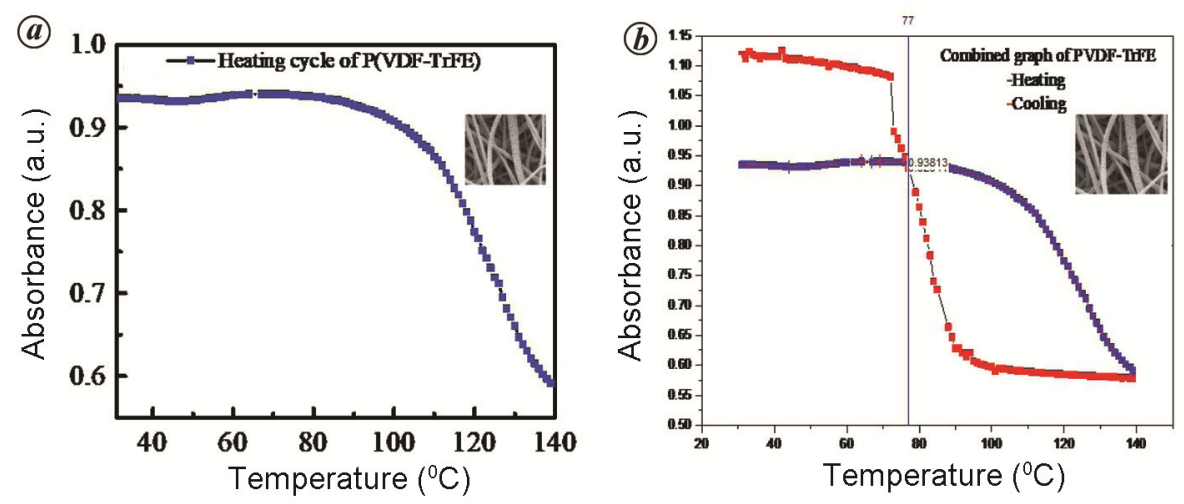

Figure 4. Fourier-transform infrared reflection (FTIR) plot of PVDF-TrFE for (a) heating cycle and (b) combined plot of the heating-cooling cycle (blue line - heating cycle; red curve - cooling phase of the film).

The crystal lattice of PVDF-TrFE is usually associated based on the molar ratio of $\mathrm{VDF} / \mathrm{TrFE}$ and also for the annealing process. The orifice in the fabricated film was perceived to play an important role in the output response of the film. Without pores, the sensitivity of the device from acoustic signals cannot rely on a reasonable level, which is distinguished using PVDF film alone without any copolymer doping (Supplementary Figure 1). The procedure to make PVDF nanofibre films has already been discussed in our previous work ${ }^{38}$. It has been shown that more pores provide a better acoustic response, which implies higher concentration doping of TrFE with PVDF. This can be elucidated by the effect of the pores on sound propagation. Direct exposure of convergent acoustic waves on nanofibres produces a higher electrical output.

The thickness of the nanofibre web also affects the output response. When the lamina of the nanofibre film is increased or wrapped with PDMS, the device sensitivity increases significantly. Output sensitivity of the sensor varies with electrode materials, in this case it shows better response.
Use of two different materials as electrodes furnishes better sensitivity over the same type of electrode (Table 1), as a potential difference between two different electrodes provides some initial potential to the sensing system, which ultimately causes higher throughput to the sensor.

When the other parameters remain unchanged, the output voltage and sensitivity show a linear increment using the thin cover of PDMS on the sensor (Table 1). This indicates that the materials also contribute to converting sound waves into electricity by polarizing electrical charges towards the surface of the sensor.

The XRD pattern and FTIR spectrum indicate that the nanofibres mainly contain $\alpha$ and $\beta$ crystal phases. XRD measurement demonstrated in Figure $3 a$ shows the ferroelectric $\beta$ phase emergence of the films, while Figure $3 b$ clearly distinguishes the enhancement of crystal characteristics with TrFE doping.

The FTIR spectra confirmed some ferroelectric properties of the TrFE doped film (Figure $4 a$ and $b$ ). The righthanded swipe from the intersection points $(77,0.938)$ precisely represents a ferroelectric loop. The temperature 


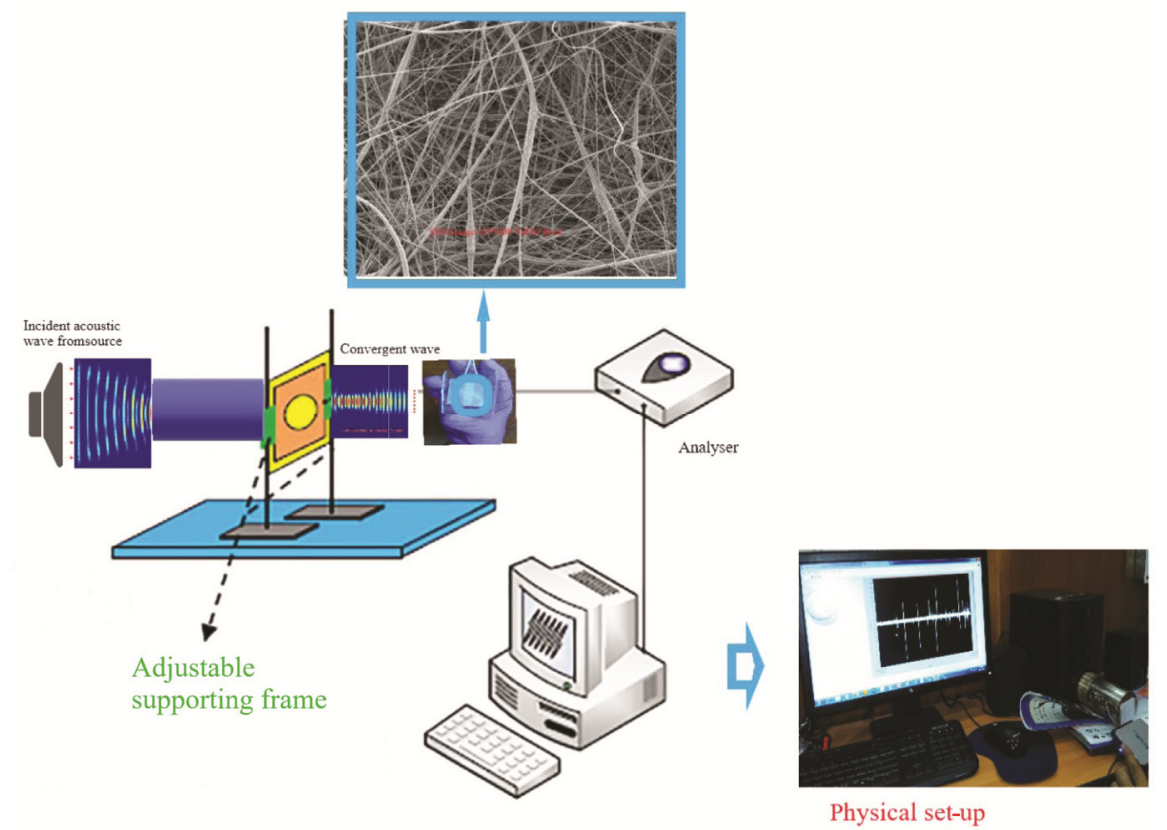

Figure 5. Schematic representation of the entire experimental set-up for signal processing along with the physical set-up for performance analysis of the fabricated sensors.

Table 1. Comparison of sensing activity of fabricated PVDF-TrFE sensors

\begin{tabular}{|c|c|c|c|c|}
\hline Sensor type & Type of input signal & Input signal (Hz) & Input signal $(\mathrm{dB})$ & Sensitivity in $\left(\mathrm{VP}_{a}^{-1}\right)$ \\
\hline $\mathrm{Al}-\mathrm{Al}$ electrode without PDMS wrapped & & & & 1.9 \\
\hline \multirow{2}{*}{$\mathrm{Al}-\mathrm{Al}$ electrode with PDMS wrapped } & & & & 6.5 \\
\hline & Normal conversation & $40-60$ & 60 & 2.1 \\
\hline Al-Cu electrode without PDMS wrapped & & & & 9.5 \\
\hline \multirow[t]{6}{*}{ Al-Cu electrode with PDMS wrapped } & Soft music & 440 & 80 & 7.5 \\
\hline & Rock music & 75 & 105 & 10.5 \\
\hline & Slow sound & 25 & 0 & 1.0 \\
\hline & Loud sound & 15,500 & 120 & 0.4 \\
\hline & Cardiac sound & $0.05-120$ & 0 & 0.01 \\
\hline & Mechanical sound & 8000 & 85 & 3.5 \\
\hline
\end{tabular}

of the intersection point is close to the Curie transition point at $73^{\circ} \mathrm{C}$, which indicates the paraelectric transformation property of the material. The asymmetry in combination with FTIR spectra between two phases (heatingcooling) provides useful information about the presence of functional groups which make the film intrinsically charged. Also, it is an indication of thermal change.

The entire experimental analysis was performed on an arbitrarily orientated nanofibre web with fibre diameter $250 \pm 30 \mathrm{~nm}$ (Figure 6). It was anticipated that fibre density and orientation will affect signal conversion. To confirm this, randomly oriented fibre webs of different film thickness were prepared. The fibres with higher thickness showed an extended number of pores, while the others had minimum pores, confirming that fibre thickness does influence sensor behaviour. All the fabricated films demonstrated acoustic to electricity conversion potential with varying piezo-potential. Under the audible sound fre- quency, thick fibre films showed better response than thin fibre films (Figure 7).

When acoustic signal incident on the nanofibers device, electric signals were generated due to conductive properties of the sensor. An instant electric pulse was generated from electron, proton and conductive ions on the sensor's surface to polarize electrical charges and create potential difference between the two electrodes. The high content of the $\beta$-crystal phase and orientation of macromolecular chain dipoles exhibit piezoelectric conversion under a compressive impact towards the nanofibers film thickness. For sound waves with frequency ranging from $30 \mathrm{~Hz}$ to $15 \mathrm{kHz}$, e.g. a normal conversation, sweet melody, rock music, whispering and telephonic conversation, the device generated a periodic output voltage within the peak value ranging from 3.50 to $11.50 \mathrm{~V}$ (Table 1). The output response was like a typical alternating current. 

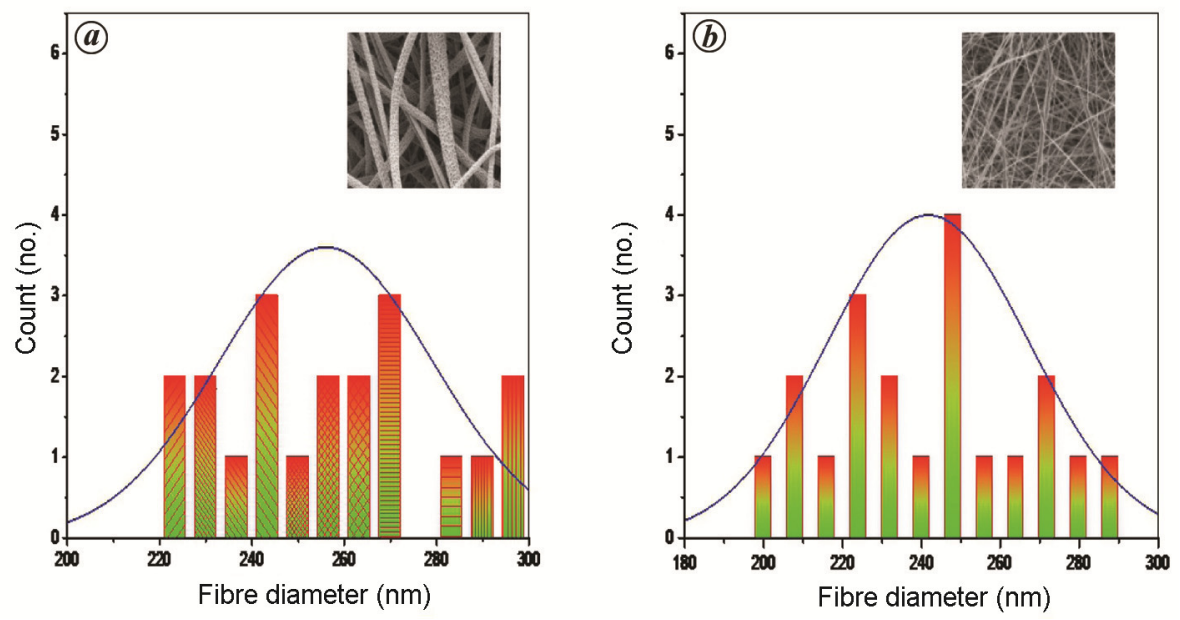

Figure 6. Diameter distribution histogram under normalized condition of (a) porous PVDF-TrFE nanofibre film and (b) PVDF nanofibres without pore.
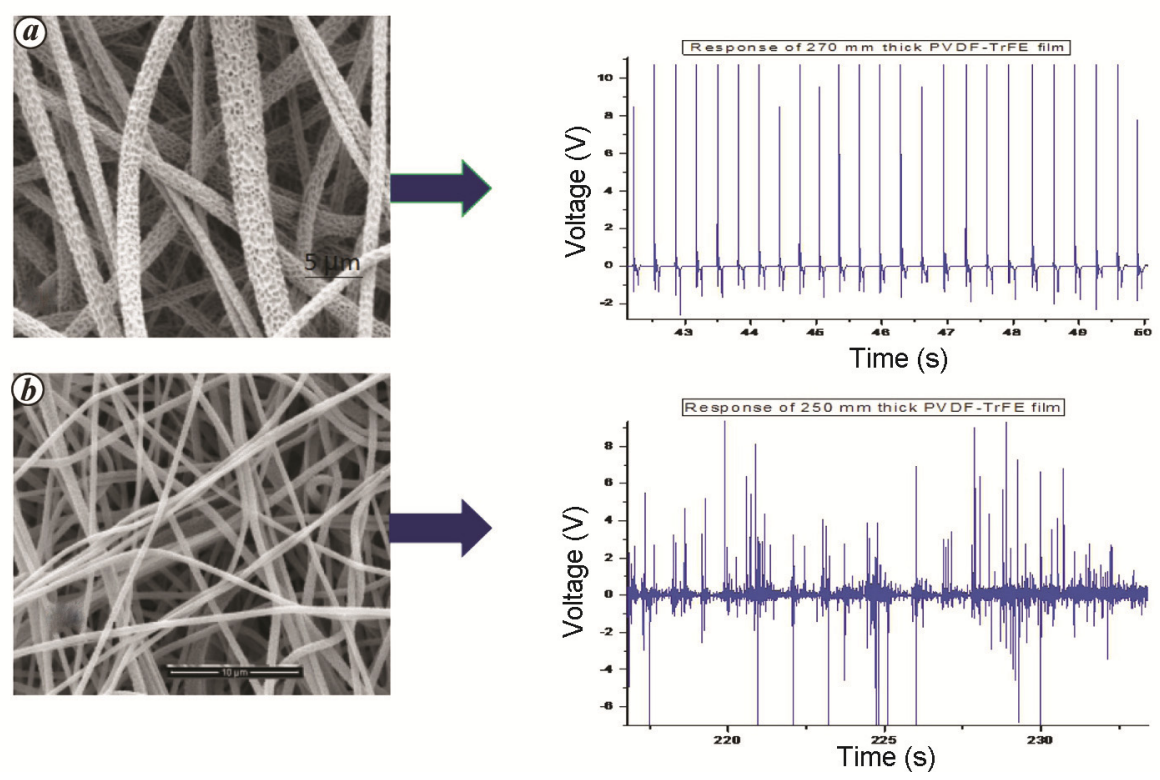

Figure 7. Output response from porous PVDF-TrFE film: $(\boldsymbol{a}) 270 \mathrm{~nm}$ thick $(\approx 11 \mathrm{~V})$ porous nanofibres film (scale bar: $5 \mu \mathrm{m})$ and $(\boldsymbol{b}) 250 \mathrm{~nm}$ thick $(\approx 9 \mathrm{~V})$ porous nanofibre film (scale bar: $10 \mu \mathrm{m})$.

To minimize noise interference from the background sound in the testing environment, electronic intermediator and connecting wires, a fast Fourier transform (FFT) and short time Fourier transform (STFT) signal processing technique was used to process the output signals. The output voltage was analysed, which was affected by acoustic wave frequency, keeping the sound pressure invariant while varying their frequencies. As shown in Figure 8 , the voltage output, signal amplitude, phase and colour distribution of the signal throughput change abruptly with incident signal frequency.

The maximum voltage output of $11.50 \mathrm{~V}$ was obtained at $3 \mathrm{kHz}$ incident acoustic signal (Supplementary Figure 2 ). When the frequency of the incident wave was above
$15 \mathrm{kHz}$ or below $30 \mathrm{~Hz}$, a very low output response was displayed (Figure $8 d 2$ and Table 1). In this range, the acoustic output was nearly zero. Signal fluctuation in the output was observed for rock music $(400-1500 \mathrm{~Hz}$, Figure $8 \mathrm{b2}$ ). Schematic diagram in normal conversation within $40-100 \mathrm{~Hz}$ resulted in nearly stable output response of $9.50 \mathrm{~V}$ (Figure $8 \mathrm{a} 2$ ). The output response of average $5.0 \mathrm{~V}$ was obtained from soft music of $440 \mathrm{~Hz}$ (Figure $8 c 2$ ).

Sensitivity is a pivotal parameter regarding the feasibility of the sensors. The sensitivity $(S)$ for acoustic sensors can be calculated as follows ${ }^{1,41}$

$$
S=V / P=V / P_{0} 10^{L_{\mathrm{p}} / 20}
$$



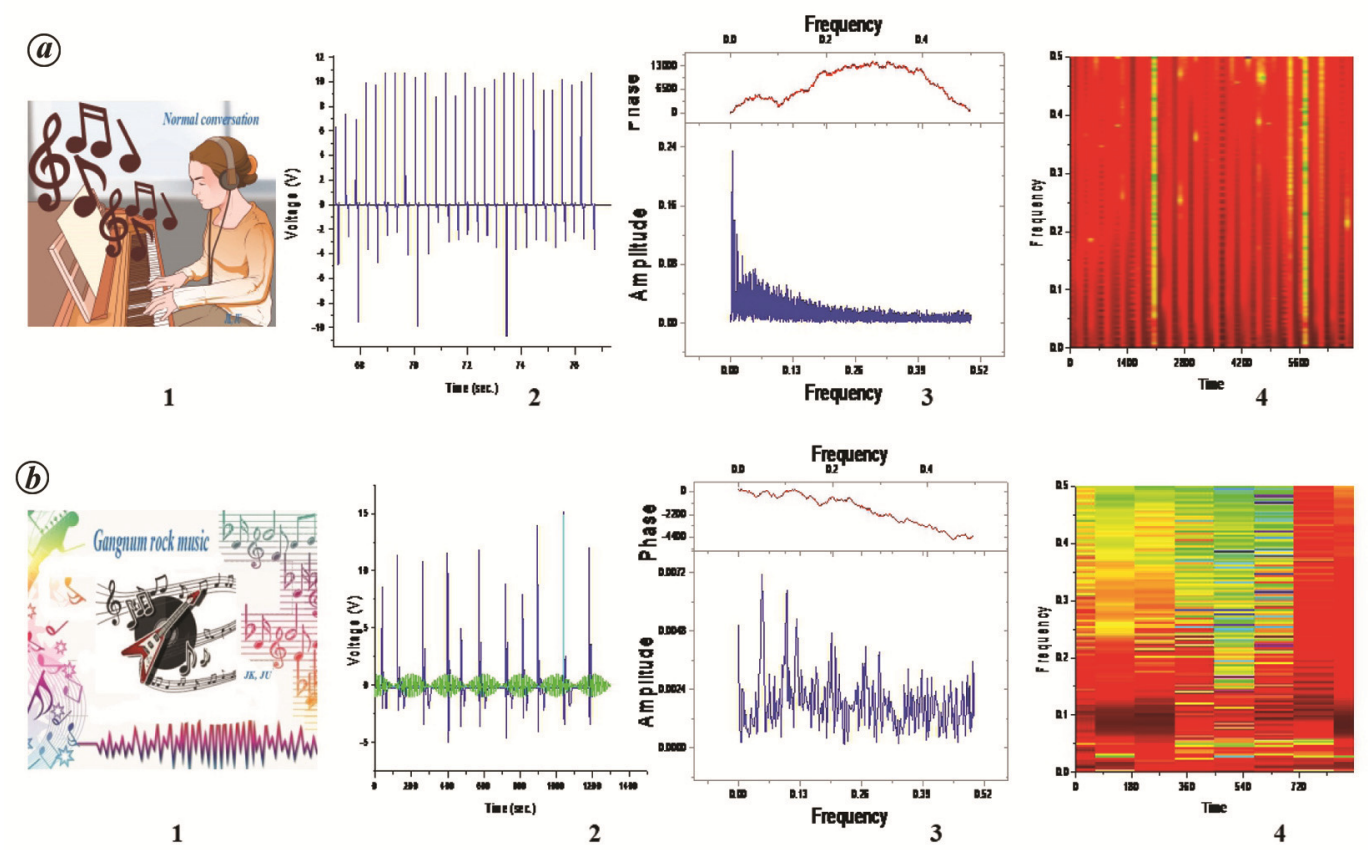

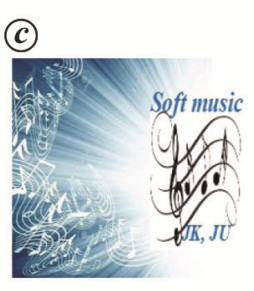

1

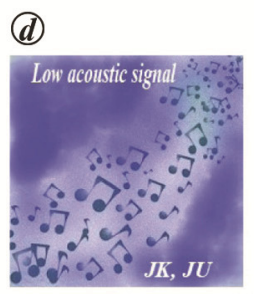

1
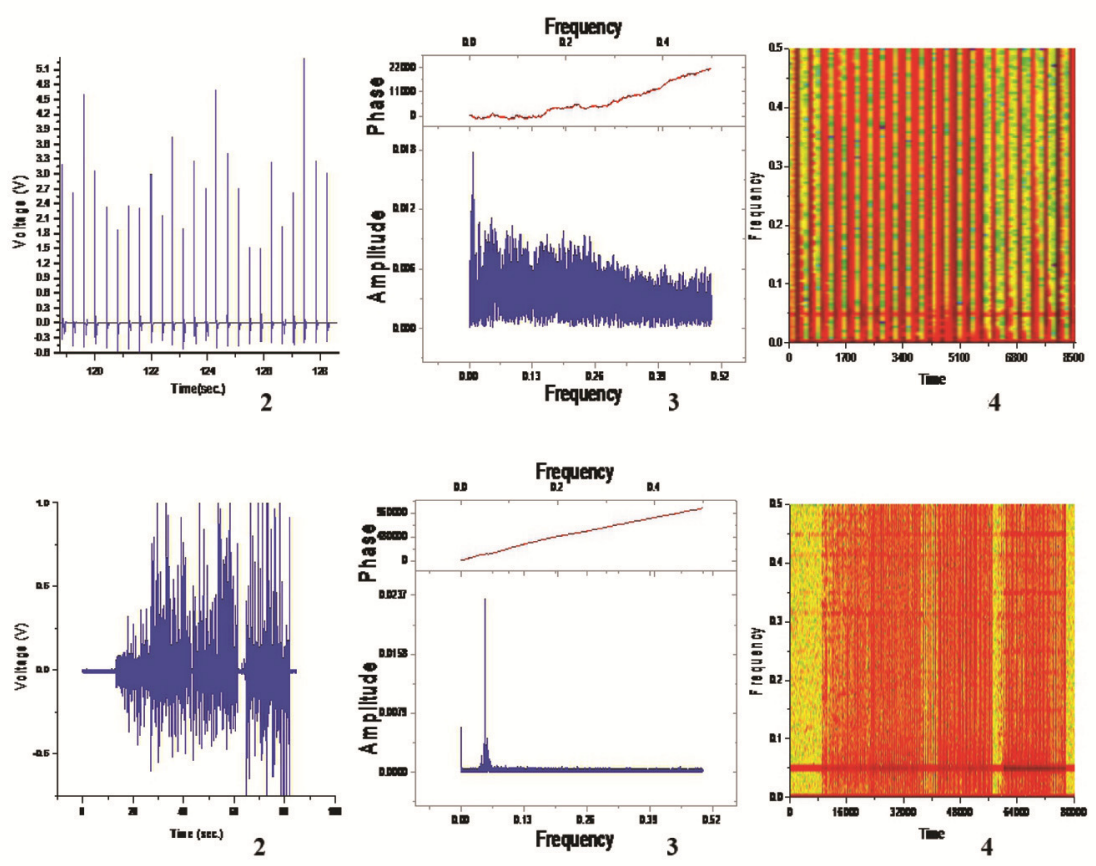

Figure 8. Output response in voltage $(\boldsymbol{a} \mathbf{2}, \boldsymbol{b 2}, \boldsymbol{c 2}$ and $\boldsymbol{d} \mathbf{2})$ of the fabricated PVDF-TrFE sensor and its fast Fourier transform in amplitude and phase $(a \mathbf{3}, b 3, c 3$ and $d \mathbf{3})$ with respect to time along with short time Fourier transform $(a \mathbf{4}, \boldsymbol{b 4}, \boldsymbol{c 4}$ and $d \mathbf{4})$ analysis in colour maps from the incident signal: (a1) normal conversation at $40-60 \mathrm{~Hz},(\boldsymbol{b} \mathbf{1})$ rock music, (c1) soft music and (d1) low acoustic signal.

where $P$ is the sound pressure, $V$ the voltage output of the device, $P_{0}$ the reference sound pressure of $0.00002 \mathrm{~Pa}$ and $L_{\mathrm{p}}$ is the SPL in decibel. In this study keeping $P$ constant, the sensitivity of the fabricated device became equivalent to the output voltage response. Using this sensor, cardiac response within the frequency range $0.05-120 \mathrm{~Hz}$ at $0 \mathrm{~dB}$ was detected with a sensitivity of $10 \mathrm{mV}$ (Figure $9 a$ ). The signal peak clearly represents QRS complex with the conventional ECG analogous of cardiac peak (Figure $9 \mathrm{~b})$.

Table 1 provides a comparison of sensing activity of the fabricated PVDF-TrFE sensors.

The PVDF-TrFE porous nanofibre acoustic sensor is flexible, but a change of shape due to flexibility may cause variation in output response. However, device response caused by input of acoustic signals is initially 

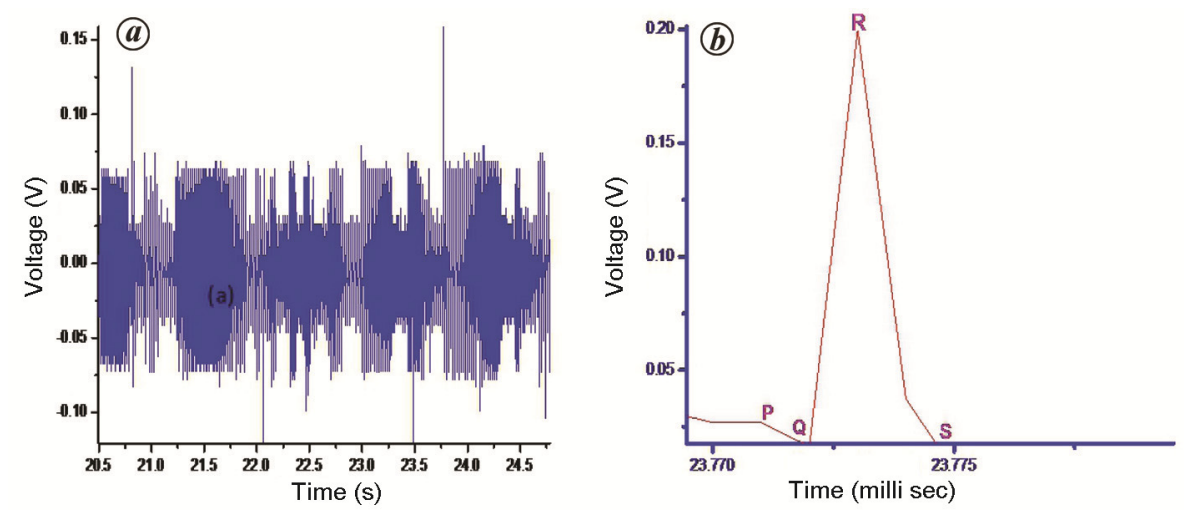

Figure 9. Output response of porous PVDF-TrFE nanofibre sensor. $\boldsymbol{a}$, Normal cardiac sound (sensor clamped on the surface of a stethoscope). $\boldsymbol{b}$, Enlarged view of ECG equivalent output peak of the sensor in terms of voltage.

localized, and variation caused by a random incident vibration arises due to change in sound frequency on the orifice of the sensor. Pores within the pore of nanofibres may cause an effective change in the output characteristics of the sensor assembly under mechanical deformation in these spans of the whole fibrous structure. PDMS encapsulation on the device provides extra advantages like protection of the main film, enhancement of overall output voltage and makes the assembly in a particular shape as per requirement. Under high temperature, the device may cause an anomaly in properties as the material undergoing non-uniform thermal stress ceases the normal behavior of the molecules. In similar conditions, the nano-fibres were produced several times with alike characteristics for other experimental purposes which confirms the high repeatability of this entire experiment.

The fabricated device successfully demonstrated acoustic to electricity conversion ability within the audible sound frequency range. The pore diameter of the nanofibre film is a significant parameter in the output response of the device. Porous film acoustic sensor devices offer better sensitivity than piezoelectric PVDF films and provide excellent ability to differentiate different sound waves in various frequency regions. Since the device distinguishes cardiac sound, a radial pulse at $0.05-120 \mathrm{~Hz}$ and $0 \mathrm{~dB}$ with significantly high sensitivity, it can be used to fabricate a sensor-based portable cardiac device. The sensor may also be used for developing various acoustic sensor-based portable, wearable and remotecontrolled biomedical devices.

Competing interest. The authors declare no competing interests.

1. Davis, D. and Patronis, E., Sound System Engineering, Focal Press, 2006, 3rd edn, pp. 420-452.

2. Hefrick Albert, D. and Cooper William, D., Modern Electronic Instrumentation and Measurement, Prentice Hall of India, 2009, 11th printing, pp. 120-137.

3. Carr Joseph, J. and Brown John, M., Introduction to Biomedical Equipment Technology, Pearson Publication, 2010, 4th edn, pp. $57-69$.
4. Khandpur, R. S., Hand Book of Biomedical Instrumentation, Tata McGraw Hill Publication, 2003, 2nd edn, pp. 22-54.

5. Blumstein Daniel, T. et al., Acoustic monitoring in terrestrial environments using microphone array. J. Appl. Ecol., 2011, 48, $758-771$.

6. Felix, A., Melkote, S. N. and Matsumoto, Y., Modeling of the holding force in an electromagnetic chuck. J. Manuf. Sci. Eng. Trans. ASME, 1999, 122(3), 366-371.

7. Thompson, L. H. and Doraiswamy, L. K., Sonochemistry: science and engineering. Sonochem. Sci., 1999, 105-112; doi.org/ 10.1021/ie9804172.

8. Sekhar, S. C., A generalized sampling method for finite-rate-ofinnovation-signal reconstruction. IEEE Signal Process. Lett., 2008, 15, 813-816.

9. Chopra, K. N. and Maini, A. K., Thin films and their application in military and civil sectors. DRDO Monogr., 2010, 402-413; ISBN 978-81-86514-32-0.

10. Beiser, A., Mahajan, S. and Choudhury, S. R., Concept of Modern Physics, SIE Publication, 2003, 6th edn, pp. 96-112.

11. Pilai, S. O., Solid State Physics, New Age Publication, 2009, 6th edn, pp. 209-216.

12. Streetman Ben, G. and Banerjee, S. K., Solid State Electronic Device, PHI Publication, 2006, 6th edn, pp. 72-87.

13. Xu, J., Dapino, M. J., Gallego Perez, D. and Hansford, D., Microphone based on polyvinylidene fluoride (PVDF) micro-pillars and patterned electrodes. Sens. Actuat. A, 2009, 153, 24-38.

14. Kim, J., High-sensitivity acoustic sensors from nanofibre webs. Report No. AOARD-084035, Inha University, 2009.

15. Consales, J., Ricciardi, A., Crescitelli, A., Esposito, E., Cutoloand, A. and Cusano, A., Lab-on-fiber technology: toward multifunctional optical nanoprobes. ACS Nano, 2012, 6(4), 3163-3170.

16. Lang, C., Fang, J., Shao, H., Dinga, X. and Lin, T., Electrospun nano-nonwoven acoustic sensors. Mater. Today Proc., 2017, 4(4), $5306-5311$.

17. Hwang, G.-T. et al., Self-powered cardiac pacemaker enabled by flexible single crystalline PMN-PT piezoelectric energy harvester. Adv. Mater., 2014, 26(28), 4880-4887.

18. Jin, K. K., Min, C. Y., Yoon, S. and Jeong, K. H., A novel piezoelectric PVDF film-based physiological sensing belt for a complementary respiration and heartbeat monitoring system. Integr. Ferroelectr., 2009, 107(1), 53-68.

19. Lovinger, A. J., Ferroelectric polymers. Ferroelectr. Polym. Sci., 1983, 220(46), 1115-1121.

20. Chang, C., Tran, V. H., Wang, J., Fuh, Y. K. and Lin, L., Directwrite piezoelectric polymeric nanogenerator with high energy conversion efficiency. Nano Lett., 2010, 10(2), 726-731.

21. Hansen, B. J., Liu, Y., Yang, R. and Wang, Z. L., Hybrid nanogenerator for concurrently harvesting biomechanical and biochemical energy. ACS Nano, 2010, 4(7), 3647-3652. 
22. Fang, J., Wang, X. and Lin, T., Electrical power generator from randomly oriented electrospun poly(vinylidene fluoride) nanofibremembranes. J. Mater. Chem., 2011, 21(30), 10881099.

23. Bauer, S., Gerhard-Multhaupt, R. and Sessler, G. M., Ferroelectrets: soft electroactive foams for transducers. Phys. Today, 2004, 57, 37-46.

24. Ramaratnam, A. and Jalili, N., Reinforcement of piezoelectric polymers with carbon nanotubes: pathway to next-generation sensors. J. Intell. Mater. Syst. Struct., 2006, 17, 199-208.

25. Furukawa, T., Lovinger, A. J., Davis, G. T. and Broadhurst, M. G., Dielectric hysteresis and nonlinearity in a 52/48 molcopolymer of vinylidene fluoride and trifluoroethylene. J. Macromol., 1983, 16(12), 1885-1890.

26. Jin, K. K. and Ling, H. S., An infra-red spectroscopic study of structural reorganization of a uniaxially drawn $\mathrm{VDF} / \mathrm{TrFE}$ copolymer in an electric field. J. Macromol., 1994, 35(17), 3612-3618.

27. Xu, H., Cheng, Z. Y., Olson, D., Mai, T., Zhang, Q. M. and Kavarnos, G., Ferroelectric and electromechanical properties of poly(vinylidene-fluoride-trifluoroethylene-chlorotrifluoroethylene) terpolymer. J. Appl. Phys., 2001, 78(16), 2360-2362.

28. Jin, K. K. and Thein, K., Spinodal phase separation and isothermal crystallization behavior in blends of VDF/TrFE(75/25) copolymer and poly(1,4-butylene adipate) (I). Fibers Polym., 2003, 4(4), 188-194.

29. Nazir, N. A., Kim, N., Iglesias, W. G., Jakli, A. and Kyu, T., Conductive behavior in relation to domain morphology and phase diagram of Nafion/poly(vinylidene-co-trifluoroethylene) blends. Polymer, 2012, 53, 196-212.

30. Li, B., Xu, C., Zheng, J. and Xu, C., Sensitivity of pressure sensors enhanced by doping silver nanowires. Sensors, 2014, 14(6), 9889-9899.

31. Roh, Y., Varadan, V. V. and Varadan, V. K., Characterization of all the elastic, dielectric, and piezoelectric constants of uniaxially oriented poled PVDF films. IEEE Trans. Ultrason., Ferroelectr. Freq. Control, 2002, 49, 836-847.

32. Sharma, T., Je, S., Gill, B. and Zhang, J. X. J., Patterning piezoelectric thin film PVDF-TrFE based pressure sensor for catheter application. Sens. Actuat. A, 2012, 177, 87-92.

33. Koga, K. and Ohigashi, H., Piezoelectricity and related properties of vinylidene fluoride and trifluoroethylene copolymers. J. Appl. Phys., 1986, 59(6), 2142-2150.

34. Higashihata, Y., Sako, J. and Yagi, T., Dynamics of ferroelectric phase transition in vinylidene fluoride/trifluoroethylene (VF2/ F3E) copolymers. I. Acoustic study. J. Ferroelectr., 1981, 90(11), 6730-6739.

35. Graz, I. et al., Flexible active-matrix cells with selectively poled bifunctional polymer-ceramic nanocomposite for pressure and temperature sensing skin. J. Appl. Phys., 2009, 106, 034503.

36. Dodds, J. S., Meyers, F. N. and Loh, K. J., Piezoelectric characterization of PVDF-TrFE thin films enhanced with $\mathrm{ZnO}$ nanoparticles. IEEE Sens. J., 2012, 12, 1889-1890.

37. Donelan, J. M. et al., Biomechanical energy harvesting: generating electricity during walking with minimal user effort. J. Sci., 2008, 319(58), 807-810.

38. Cheng, Z. Y., Bharti, V., Xu, T. B., Haisheng, Xu, Mai, T. and Zhang, Q. M., Electrostrictive poly(vinylidene fluoride-tri fluoroethylene) copolymers. Sens. Actuat. A, 2001, 90, 138147.

39. Ramadan, K. S., Sameoto, D. and Evoy, S., A review of piezoelectric polymers as functional materials for electromechanical transducers. Smart Mater. Struct., 2014, 23, 26-32.

40. Kibria, F. and Patra, S. N., Fabrication of fiber aided nano device for minimally invasive photonics based sub-micron level lesion detection and interventional treatment. IJREAM, 2018, 4(8), 478484 .
41. Park, T., Kim, B., Kim, Y. and Kim, E., Highly conductive PEDOT electrodes for harvesting dynamic energy through piezoelectric conversion. J. Mater. Chem., 2014, 232-241; doi.org/ $10.1039 / \mathrm{c} 3$ ta14726f.

42. Toda, M. and Thompson, M. L., Contact-type vibration sensors using curved clamped PVDF film. IEEE Sens., 2006, 6(5), 1170 1177 .

ACKNOWLEDGMENTS. We thank the staff of the ONPDL Lab, Department of Physics, Jadavpur University for technical support. The work was funded by the Departmental Internal Grand Fund for Research of Excellence under Jadavpur University. Permission to collect samples for experimental work was undertaken by the central facilities under Jadavpur University, Kolkata.

Received 24 August 2019; revised accepted 8 January 2020

doi: $10.18520 / \mathrm{cs} / \mathrm{v} 119 / \mathrm{i} 5 / 841-849$

\section{New record of marine red algal species Grateloupia orientalis Showe M. Lin \& H.Y. Liang and G. catenata Yendo (Halymeniaceae, Rhodophyta) from the east coast of India}

\author{
P. Chellamanimegalai, Annam Pavan-Kumar, \\ Amjad K. Balange, A. Dwivedi and \\ Geetanjali Deshmukhe* \\ ICAR-Central Institute of Fisheries Education, Panch Marg, \\ Off Yari Road, Versova, Andheri West, Mumbai 400 061, India
}

The present study reports the new record of two Grateloupia species (Halymeniales, Rhodophyta) namely Grateloupia orientalis Showe M. Lin \& H.Y. Liang and G. catenata Yendo from the coast of Visakhapatnam in Andhra Pradesh, and Tiruchendur in Tamil Nadu, India respectively. Morphological and anatomical studies showed that these differed from the earlier recorded Grateloupia species, viz. G. indica, G. filicina and $G$. lithophila. The descriptive statistics and MDS analysis revealed that these two species differed from the earlier recorded Grateloupia sp. The taxonomic evaluation of these two Grateloupia species based on chloroplast-encoded $r b c L$ gene revealed that they are closely related, sharing few traits between them.

Keywords: Coastal regions, descriptive statistics, invasive species, marine red algae, taxonomic evaluation.

THE Rhodophycean algae Grateloupia (C. Agardh 1822) is one of the largest genera of family Halymeniaceae with

*For correspondence. (e-mail: dgeetanjali@cife.edu.in) 